\title{
Hepatic PKA inhibition accelerates the lipid accumulation in liver
}

Jining Yang ${ }^{1}$, Xiaoying Zhang ${ }^{2}$, Long $\mathrm{Yi}^{1}$, Ling Yang ${ }^{3}$, Wei Eric Wang ${ }^{4}$, Chunyu Zeng ${ }^{4}$, Mantian $\mathrm{Mi}^{{ }^{*}}$ and Xiongwen Chen $^{2^{*}}$

\begin{abstract}
Background/aims: Liver lipid accumulation induced by high-fat diet (HFD) is an early onset process of nonalcoholic fatty liver diseases (NAFLD). Protein kinase A (PKA) is known to be involved in hepatic lipid metabolism. However, the role of PKA in NAFLD has not been well tested in vivo due to the lack of optimal PKA deficient mouse model.

Methods: A novel PKA-specific inhibitor gene was conditionally overexpressed in mouse (PKAi mouse) liver using LoxP/Cre system. PKA activity in the liver extract was measured with a commercial assay kit. The PKAi and control mice of 8-week age, were subjected to HFD or chow diet (CD) for 2 months. Body weight, liver index, and triglyceride in the liver were measured. RNA sequencing was performed for the liver tissues and analyzed with Gene Ontology (GO) and pathway enrichment.
\end{abstract}

Results: PKAi-GFP protein was overexpressed in the liver and the PKA activation was significantly inhibited in the liver of PKAi mouse. When fed with CD, RNA sequencing revealed 56 up-regulated and 51 down-regulated genes in PKAi mice compared with control mice, which were mainly involved in lipid metabolism though no significant differences in the body weight, liver index, triglyceride accumulation were observed between PKAi and control mice. However, when fed with HFD for 2 months, the liver was enlarged more, and the accumulation of triglyceride in the liver was more severe in PKAi mice. When comparing the transcriptomes of CD-fed and HFD-fed control mice, GO enrichment showed that the genes down-regulated by HFD were mainly enriched in immune-related GO terms, and up-regulated genes were enriched in metabolism. When comparing the transcriptomes of CD-fed and HFD-fed PKAi mice, GO analysis showed that the down-regulated genes were enriched in metabolism, while the up-regulated genes were clustered in ER stress-related pathways. When comparing HFD-fed PKAi and HFD-fed control mice, the genes with lower expression level in PKAi mice were enriched in the lipoprotein synthesis, which might explain that more TG is accumulated in PKAi liver after HFD feeding.

Conclusions: Reduced PKA activity could be a factor promoting the TG accumulation in the liver and the development of NAFLD.

Keywords: Protein kinase a, Non-alcoholic fatty liver diseases, Lipid metabolism, RNA sequencing

\footnotetext{
* Correspondence: mi_mt2009@hotmail.com; xchen001@temple.edu

${ }^{1}$ Research Center for Nutrition and Food Safety, Chongqing Key Laboratory

of Nutrition and Food Safety, Institute of Military Preventive Medicine, Third

Military Medical University, Chongqing, People's Republic of China

${ }^{2}$ Department of Physiology \& Cardiovascular Research Center, Temple

University Lewis Katz School of Medicine, Philadelphia, PA, USA

Full list of author information is available at the end of the article
}

(c) The Author(s). 2019 Open Access This article is distributed under the terms of the Creative Commons Attribution 4.0 International License (http://creativecommons.org/licenses/by/4.0/), which permits unrestricted use, distribution, and reproduction in any medium, provided you give appropriate credit to the original author(s) and the source, provide a link to the Creative Commons license, and indicate if changes were made. The Creative Commons Public Domain Dedication waiver (http://creativecommons.org/publicdomain/zero/1.0/) applies to the data made available in this article, unless otherwise stated. 


\section{Background}

Nonalcoholic fatty liver disease (NAFLD) consists a wide pathological spectrum ranging from steatosis, nonalcoholic steatohepatitis (NASH), fibrosis, and cirrhosis to hepatocellular carcinoma; and has become the fastest growing liver disease worldwide with a prevalence as high as a $25 \%$ of the population. NAFLD threatens the public health and causes a huge economic burden [1, 2]. Though several studies have detected molecules as potential therapeutic targets, there is no US FDA-approved therapy for NAFLD [2]. Therefore, it is necessary to understand the underlying mechanism of this disease to meet the need for drug discovery and treatment for patient benefit.

The underlying mechanism for the development and progression of NAFLD is complex and multifactorial, among which lipid accumulation in liver is an early step $[3,4]$. There are multiple mechanisms leading to lipid accumulation in hepatocytes, including increased free fatty acids supply and de novo hepatic lipogenesis; and decreased free fatty $\beta$-oxidation in the liver as well as decreased hepatic lipid export [5]. Lipid accumulation may also result in the early "inflammatory" hit, thus leading to the whole spectrum of NAFLD pathologies [3].

Protein kinase A (PKA) plays a crucial role in the regulation of lipid metabolism. The dysregulation of PKA signaling is associated with the pathogenic mechanisms underlying many metabolic diseases, including NAFLD [6, 7]. The pharmacological studies show that the activity of PKA is linked to the pharmacological effects of the major lipid regulating agents [8-12]. For example, the wellknown antioxidant resveratrol could reduce the lipid accumulation and alleviate NAFLD in a PKA-dependent way, suggesting that the activity of PKA might be a promising target in treating NAFLD [8]. However, the effect of activation of PKA in hepatic lipid synthesis seems to be conflicting. For example, the in vitro study showed that PKA activation by glucagon or forskolin downregulates the lipid synthesis efficiency in hepatocytes by phosphorylating cAMP response element binding protein (CREB) to increase the expression of ring finger protein-20 (RNF-20), which mediates the degradation of sterol regulatory element binding protein-1c (SREBP-1c) through ubiquitination [13]. On the other hand, PKA activation induced by thyrotropin in hepatocytes can upregulate SREBP-1c by phosphorylating peroxisome proliferators-activated receptors alpha (PPAR $\alpha)$ to promote liver lipid synthesis [14]. Therefore, it is necessary to study the role of PKA in hepatocytes in the development of NAFLD in vivo and understanding the role of PKA in TG accumulation in the liver might provide promising strategies in developing therapy for NAFLD.

PKA is widely distributed in almost every type of cells of mammals, with a highly conserved structure among species [15]. The structure of PKA was first revealed in 1991 [16] as a heterotetramer consisting of 2 regulatory (PKA R) subunits that have 4 isotypes (RI $\alpha, \mathrm{RII} \alpha, \mathrm{RI} \beta$, $\mathrm{RII} \beta$ ) and 2 catalytic subunits (PKA C) that have 4 isotypes $(\mathrm{C} \alpha, \mathrm{C} \beta, \mathrm{C} \gamma, \operatorname{Prkx})$. The catalytic subunits are bound to the regulatory subunit dimer and stay inactive when cAMP level is low, because the regulatory subunit contains a pseudosubstrate domain to inhibit the kinase activity of the catalytic subunit [17]. When cytosolic cAMP is increased, the PKA R binds to cAMP, causing a conformational change that decreases its affinity for catalytic subunits and leads to the release of catalytic subunits, which then become active and phosphorylate serine and threonine in its target proteins. The activation of PKA plays a crucial role in lipolysis through many PKA targets involved in lipid metabolism, such as hormone sensitive lipase (HSL) [18]. Due to the existence of various isotypes of the subunits of PKA encoded by multiple genes in the same cell, it remains almost impossible to generate a hepatocyte-specific complete PKA knockout animal model to explore its roles in hepatic lipid metabolism in vivo. Genetically, to knockout all three PKA catalytic genes is very difficult. There is no PKA specific inhibitor neither [19] and PKA inhibition drug cannot achieve tissue specificity. For example, the widely used classical PKA inhibitor H89 can partially inhibit other proteins such as protein kinase B (PKB) [19]. Thus, it remains unclear whether hepatic PKA promotes or inhibits lipid accumulation in vivo. To circumvent this problem, we took advantage of the Cre/LoxP system [20] and constructed a transgenic mouse model that would overexpress a designed PKA inhibition (PKAi) fusion gene, containing the nucleotide sequence coding the amino acids $1-25$ of PKA inhibitor peptide (PKI)- $\alpha$ and green fluorescent protein (PKAi-GFP) [21] specifically in the liver. PKAi contains the PKA inhibition domain of PKI- $\alpha$, a naturally coded protein. PKAi, as a pseudosubstreate, interacts with the peptide/protein binding portion of the active site of the catalytic subunit in a competitive manner with a low nanomolar Ki value, thus inhibiting the activity of PKA [22].

After confirming the hepatic inhibition of PKA in the mouse model, we studied the body weight, liver index and triglyceride accumulation in the liver of mice fed with chow diet (CD) or high-fat diet (HFD). Also, unbiased RNA sequencing was done to elucidate the differences in transcription upon the inhibition of PKA in the liver of mice fed with normal or HFD chow. We found that PKA inhibition aggregates triglyceride accumulation in the liver after HFD feeding. Our findings provided new direct evidence regarding the role of PKA in lipid metabolism in vivo, and elucidated the mechanisms based on transcriptome analysis, which might open new avenues to combat NAFLD. 


\section{Methods}

\section{Experimental animals and treatments}

Establishment of PKAi-GFP transgenic mice: A DNA sequence coding a peptide (animo acids sequence: TDVETTYADFIASGRTGRRNAIHD) of the PKA inhibitory domain of mouse protein kinase inhibitor $\alpha$ (mouse Entrez gene ID 18767) was synthesized and subcloned into plasmid pAcGFP1-N1 (Clontech) to make a PKAiGFP fusion gene. Then this fusion gene was subcloned into the CAG-LoxP-CAT-LoxP vector provided by Dr. Qinglin Yang (Louisianan State University). Then the transgenic mouse line carrying CAG-LoxP-CAT-LoxPPKAi-GFP was made by the Institute of Model Animal of Wuhan University. In this transgenic mouse line, the expression of PKAi-GFP was blocked by a stop sequence (a bacteria chloramphenicol, acetyltransferase gene, CAT) [23]. This mouse line was crossbred with the hepatocyte-specific cre (Alb-cre) homozygous mouse line to obtain the double transgenic (CAG-LoxP-CATLoxP-PKAi-GFP/Alb-cre) mice. In hepatocytes of the double transgenic mice, CAT was excised by cre, and then PKAi-GFP expression was induced. Littermate mice with only a single transgene (Alb-cre or CAG-LoxP-
CAT-LoxP-PKAi-GFP) and with matched age and sex were used as controls. (Fig. 1a).

Treatments: male PKAi mice $(n=12)$ and male control mice $(n=12)$ were fed with CD till 8-week old, and then divided randomly into four groups (6 mice/group): PKAi mice fed with CD (PKAi_CD), PKAi mice fed with HFD (PKAi_HFD), control mice fed with CD (control_CD) and control mice fed with HFD (control_HFD). Mice were then fed with either CD (10\% calories from fat, D12350B, Research Diets, US) or HFD (45\% calories from fat, D12451, Research Diets, US) for 2 months. Detailed information of the diets was displayed in Additional file 1. All animals were maintained under a regular 12-h light/12-h dark period at a controlled temperature $\left(22 \pm 2{ }^{\circ} \mathrm{C}\right)$ and humidity $(50 \pm 5 \%)$, and received food and water ad libitum. Body weight and diet consumption were recorded weekly during the study. Liver samples of the same region of left lobe of each mice were collected for the following experiments. Animal care and treatments were conducted according to established guidelines and protocols approved by the Animal Care and Use Committee of the Third Military Medical University.

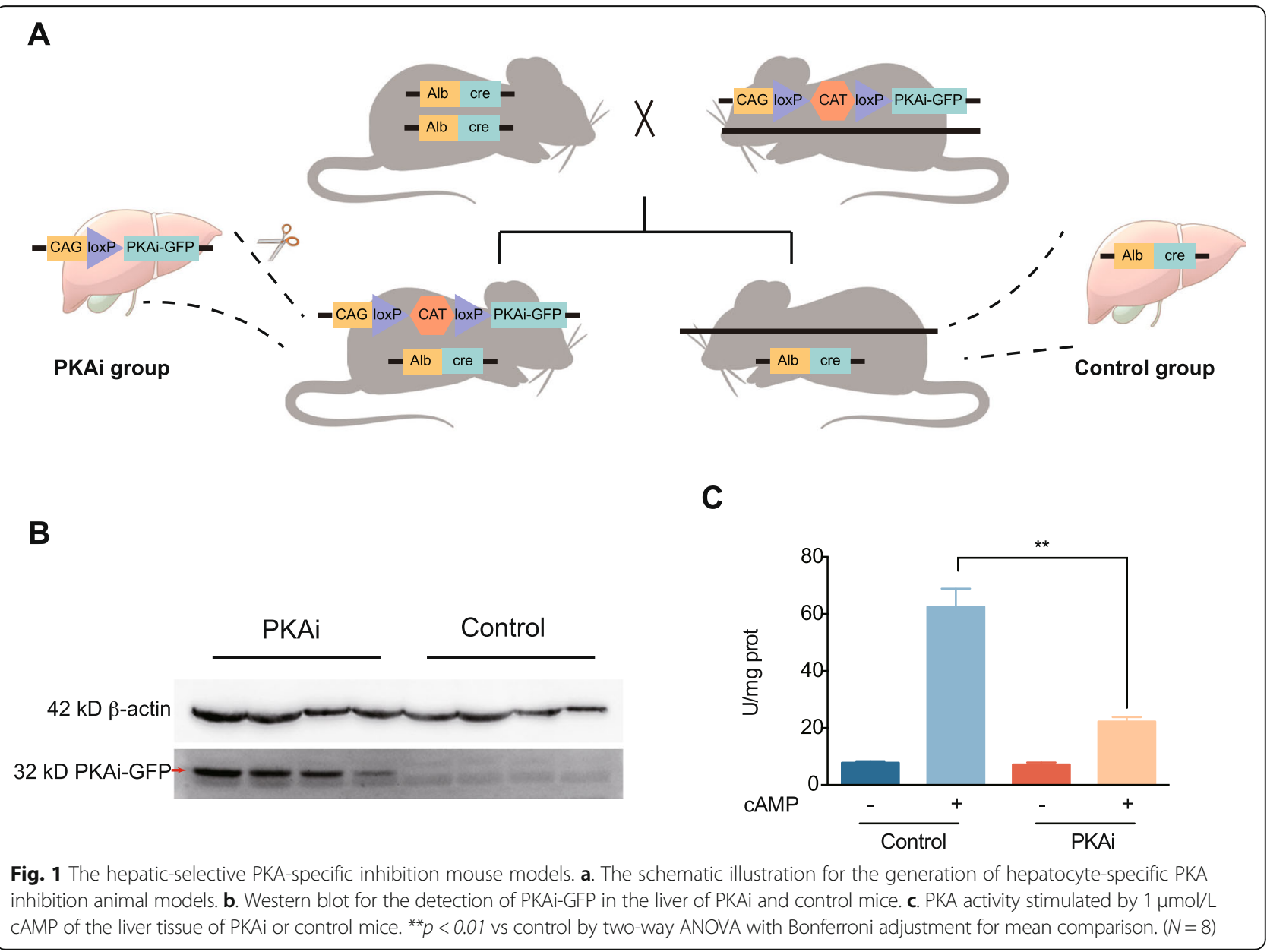




\section{Western blot}

Whole protein samples extracted by T-PER lysis buffer (Thermo Fisher Scientific Inc., US) from liver tissues of 4. PKAi and 4 control mice (male, 8-week old) were used to determine the abundance of PKAi-GFP with anti-GFP antibody (Abcam, USA) by standard Western blotting protocol. $\beta$-actin (Cell Signaling Technology, USA) was used as the loading control. Briefly, equal amounts $(20 \mu \mathrm{g})$ of proteins were subjected to $10 \%$ sodium dodecyl sulfate polyacrylamide gel electrophoresis and then electroblotted onto polyvinylidene difluoride membranes, which were subsequently blocked with TBST containing 5\% BSA for $1 \mathrm{~h}$ before probed with primary antibodies overnight at $4{ }^{\circ} \mathrm{C}$. Membranes were then incubated with a secondary anti-rabbit antibody (Cell Signaling Technology, USA), and the immunostained bands were visualized with Immobilon Western Chemiluminescent HRP Substrate (Millipore, USA) and captured by a Fusion FX (Vilber Lourmat, France) machine. The antibody information was displayed in Additional file 2.

\section{RNA isolation and quantitative real-time polymerase chain reaction (qRT-PCR)}

To determine PKAi-fusion gene expression, various tissues of the PKAi mice (8-week old) were harvested in RNAiso Plus reagent (Takara Bio, Japan), and the total RNA was extracted according to the manufacturer's instructions. To prevent the genomic DNA contamination, we treated the RNA sample with TURBO DNA-free kit (Invitrogen, US). Then the RNA concentration and purity were measured by NanoDrop 2000 spectrophotometer (Thermo Fisher Scientific Inc., US). The first-strand cDNA was synthesized by a PrimeScript RT Master Mix kit (Takara Bio, Japan). qRT-PCR was carried out with the qTower 2.2 real-time PCR system (Anakytik Jena, Germany) using SYBR Premix Ex Taq II (Takara Bio, Japan). The primers for the targeted genes were synthesized by Sangon Biotech (Shanghai, China). The primer sequences used for gene expression analysis are listed in Additional file 3. The amplification profile consisted of denaturation at $95^{\circ} \mathrm{C}$ for $30 \mathrm{~s}$, followed by 40 cycles of $95^{\circ} \mathrm{C}$ for $5 \mathrm{~s}$ and $60^{\circ} \mathrm{C}$ for $30 \mathrm{~s}$. A melting curve stage was performed at the end of the amplification. Relative fold-changes in gene expression were analyzed by the $2^{-\Delta \Delta \mathrm{Ct}}$ method and normalized to the internal control gene $\beta$-actin [24].

\section{Measurement of PKA activity}

The control and PKAi mouse liver samples were homogenized in lysis buffer supplemented with $0.4 \mathrm{mmol} / \mathrm{L} 3-$ isobutyll-methylxanthine (IBMX, a phosphodiesterase inhibitor, Sigma-Aldrich, US). Basal PKA activity or maximal PKA activity stimulated with $1 \mu \mathrm{M}$ cAMP in the homogenates were assessed with a PKA kinase activity assay kit (Assay Design, Ann Arbor, MI), following the provided protocol. Briefly, $40 \mu \mathrm{L}$ of samples and fully active recombinant PKA standard at different concentrations were added into the wells of the plate. Next, $10 \mu \mathrm{L}$ of the reconstituted ATP was added to each of the well followed by the incubation at $30^{\circ} \mathrm{C}$ for $90 \mathrm{~min}$. After carefully aspirating the reagents, the plate was washed 4 times with $300 \mu \mathrm{L}$ wash buffer and tapped to dry on clean absorbent towel. For the antigen-antibody binding, $25 \mu \mathrm{L}$ of the Rabbit Anti-Phospho PKA Substrate Antibody and $25 \mu \mathrm{L}$ Goat anti-Rabbit IgG HRP Conjugate were added to each well. Then the plate was sealed and incubated at room temperature for $60 \mathrm{~min}$ with shaking. Afterwards, the wells were aspirated again and rinsed 4 times with wash buffer. After the final wash, $100 \mu \mathrm{L}$ of the TMB substrate solution was added to each well and incubate the plate at room temperature for $30 \mathrm{~min}$. Finally, $50 \mu \mathrm{L}$ of the stop solution was added to each well and the optical density was read by a plate reader at $450 \mathrm{~nm}$. The PKA activity was calculated from the linear standard curve using linear regression and normalized to the protein concentration.

\section{RNA extraction, library preparation and sequencing}

Mice of the four groups (PKAi_CD, PKAi_HFD, Control_CD and Control_HFD) were raised as described above. Then, 3 mice of each group were sacrificed and their liver tissues were harvested for RNA isolation using the TRIzol (Invitrogen, US) as described in the instruction. RNA quality was determined by examining A260/A280 with a Nanodrop ${ }^{\text {Tw }}$ OneCspectrophotometer (Thermo Fisher Scientific Inc., US). RNA Integrity was confirmed by $1.5 \%$ agarose gel electrophoresis. Qualified RNAs were finally quantified by Qubit3.0 with Qubit $^{\text {is }}$ RNA Broad Range Assay kit (Life Technologies, US) and used for stranded RNA sequencing library preparation using $\mathrm{KC}^{\mathrm{m}}$ Stranded mRNA Library Prep Kit for Illumina ${ }^{\bullet}$ (Wuhan Seqhealth Inc., China). PCR products corresponding to $200-500 \mathrm{bp}$ were enriched, quantified and finally sequenced on Hiseq X10 sequencer (Illumina, US).

\section{RNA-seq data analysis}

Sequencing reads that were in low quality or contained only adapters were pre-filtered and then the rest sequences were mapped to the $\mathrm{mm} 9$ genome using HISAT [25]. Clean reads were mapped to reference by featureCounts [26], and then reads per kilobase per million mapped reads (RPKMs) were calculated. We detected differentially expressed genes (DEGs) between groups with edgeR package [27]. Genes were called differentially expressed if they exhibited an adjusted $P$ value $<0.05$. Heatmap of the DEGs were generated using the heatmap $R$ package [28]. Gene ontology (GO) enrichment analysis 
of DEGs were analyzed by KOBAS software [29] and the top 10 terms (ranked by q-value, the modified Fisher exact $P$-value) were displayed by the $\mathrm{R}$ package ggplot2 [30]. Gene set enrichment analysis (GSEA) [31] was used to investigate potential mechanisms in the Molecular Signatures Database (MSigDB) [32] of h (h.all.v6.2. symbols). The enrichment gene sets in GSEA with the lowest false discovery rate (FDR) was demonstrated. Metascape online tool [33] was used to do the network analysis. Briefly, DEGs of each group that were just up/ down-regulated in control mice or just up/down-regulated in PKAi mice induced by HFD were put into two lists and uploaded to the website. Subsequently, enrichment analysis was performed online using Reactome Gene Sets [34] and Kyoto Encyclopedia of Genes and Genomes (KEGG) [35] database. Pathways with minimum overlap at $3, P$-value cutoff at 0.01 , and minimum enrichment at 1.5 were screened out and displayed in clusters. The thickness of lines between nodes represents the kappa score of overlapping genes of the linking nodes. The RNA-seq data entitled "Transcriptome studies of liver tissue in PKAi mouse model" were deposited to the Sequence Read Archive (SRA) with BioProject number PRJNA553077.

\section{Triglyceride assay}

The triglyceride level of liver samples and the intracellular triglyceride content was determined with an enzymatic assay kit (Applygen Technologies Inc., Beijing, China) as described in the kit instruction. The protein concentrations were determined with a BCA Protein Assay Kit (Beyotime, China) as described previously. The results were expressed as mmol per gram protein (mmol/g prot).

\section{Hematoxylin \& eosin (H\&E) staining}

Liver tissue was fixed in $4 \%$ paraformaldehyde, dehydrated, embedded in paraffin wax, and then sectioned into $5 \mu \mathrm{m}$-thick slices. The sections were subsequently deparaffined and rehydrated via a xylene and alcohol series before performing H\&E staining according to standard histology procedure as previously described [36].

\section{Oil red $O$ staining}

Oil Red O powder (0.5 g, Sigma-Aldrich, USA) was evenly dissolved in $100 \mathrm{~mL}$ of isopropanol to prepare stock solution, which was then diluted with distilled water at the ratio of 3:2 and filtered to make the working solution. Liver tissue cryosections $(8 \mu \mathrm{m})$ were prepared in a cryostat (Leica, Germany) and stained with prewarmed Oil Red O working solution for $15 \mathrm{~min}$. After rinsing with distilled water three times, the sections were then counterstained with hematoxylin (Sigma-Aldrich, USA) for $3 \mathrm{~min}$ and gently washed with distilled water.
Representative images were captured using a light microscopy (Leica, Germany).

\section{Statistical analysis}

Data were reported as mean \pm SEM and analyzed with SPSS 19.0 software (Chicago, USA). Statistical differences among groups were determined with Student's ttest or two-way ANOVA. $P$ values less than 0.05 were considered statistically significant.

\section{Results}

PKAi-GFP is specifically expressed in the mouse liver to suppress PKA activity

Hepatic-specific PKA inhibition mice and corresponding control mice were obtained by crossing the heterozygotes CAG-LoxP-CAT-LoxP-PKAi-GFP transgenic and Alb-cre transgenic mice (Fig. 1a). PKAi-GFP was expressed only in CAG-LoxP-CAT-LoxP-PKAi-GFP/Alb-cre double transgenic mice (termed PKAi mice here) but not in control mice, validated by western blots in mouse liver tissue (Fig. 1b). Tissue specific expression of PKAi-GFP was determined using qRT-PCR, showing that overexpressed PKAiGFP was predominantly expressed in the liver but little in other tissues (Additional file 4). There was little PKA activity at baseline in both control and PKAi mice liver. However, when crude liver tissue extract was stimulated by $1 \mu \mathrm{mol} / \mathrm{L}$ cAMP, PKA activity was significantly increased in control mouse liver samples but not as much in PKAi transgenic samples, indicating that overexpressed PKAi-GFP in PKAi mouse liver was sufficient to inhibit most of cAMP-induced PKA activity (Fig. 1c).

\section{Transcriptome analysis of liver from PKAi and control} mice showed fatty acid metabolism pathway being dominantly affected by hepatocyte-specific PKA inhibition PKA plays an important role in regulating lipid and glycogen metabolism in the liver [37]. We first performed the next generation RNA-seq to compare the transcriptomes of the livers of PKAi with those of control mice in order to find out the major functions and pathways affected by PKA inhibition in liver. One hundred and seven DEGs (51 down-regulated, and 56 upregulated in PKAi mice) were screened out by analyzing the RNA-seq data. The heatmap in Fig. 2a showed the hierarchical clustering of these DEGs, which distinguished the two groups very well. Interestingly, among these DEGs, fgf 21 was the most differentially expressed gene, down-regulated in PKAi mice with a log (foldchange) of 4.43 (Additional file 5). Fgf21 is a well-known protective factor in attenuating NAFLD by promoting free fatty acid oxidation [38]. GO enrichment analysis found 42 GO terms significantly enriched in upregulated DEGs and 35 GO terms significantly enriched in down-regulated DEGs (q-value $<0.05$ ) in PKAi mice. 


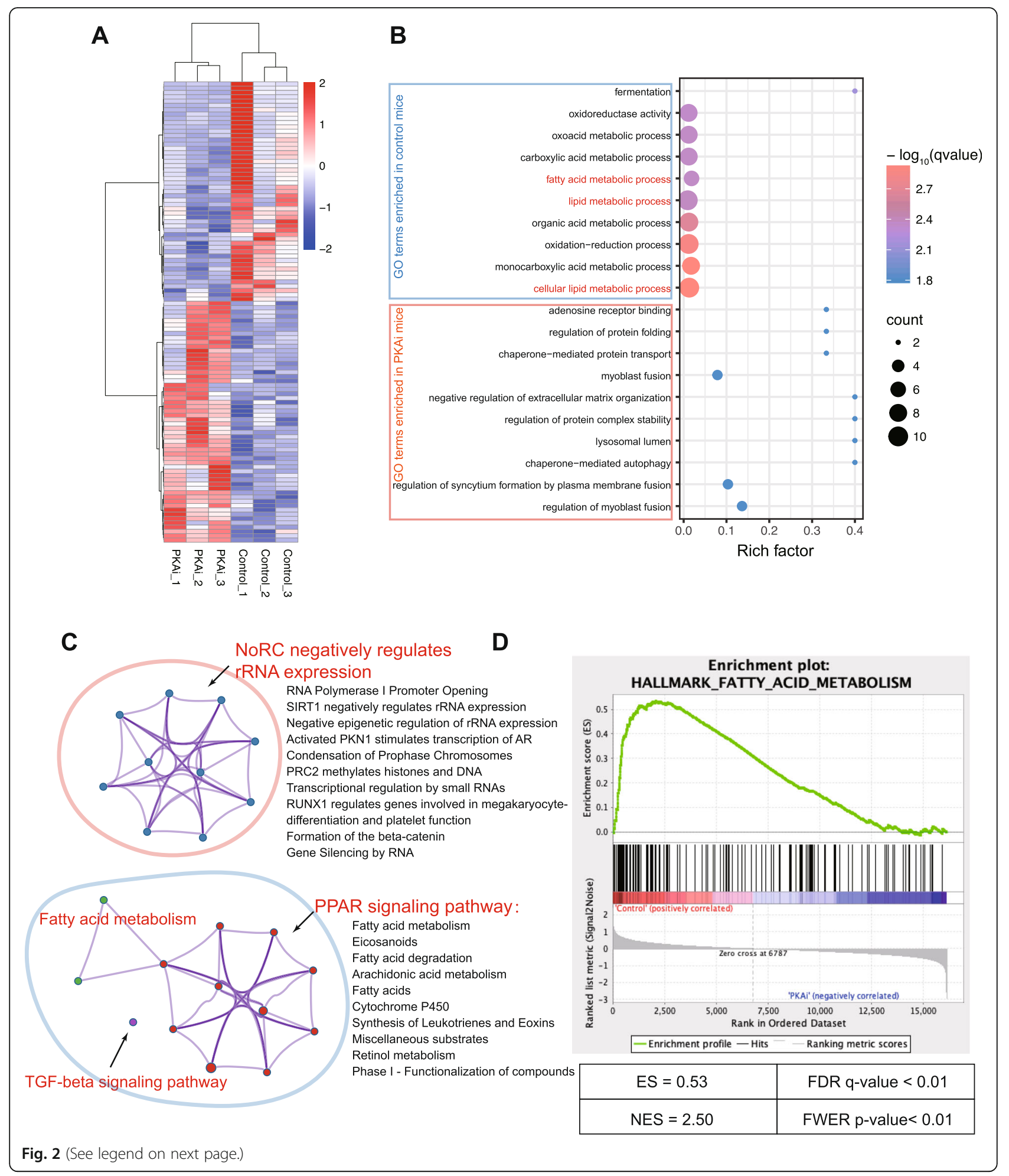


(See figure on previous page.)

Fig. 2 Transcriptome analysis of PKAi-GFP mice at baseline. Total mRNA was isolated from the livers of 3 PKAi and 3 control mice of 16-week old fed with chow diet, and then used to construct library for RNA sequencing. a The heatmap of differentially expressed genes (DEGs) of PKAi and control mice. b The top 10 enriched Gene ontology (GO) terms (ranked by q-value, i.e. a modified Fisher exact P-value) of the DEGs. Rich factor indicates the ratio of input gene number to background gene number of each term. c Clustered pathways of the DEGs based on KEGG and Reactome database. Each node represents a pathway, and the nodes in one color represent a cluster of pathways. The nodes in red circle were the enriched pathways of up-regulated DEGs, and the nodes in blue circle were the enriched pathways of down-regulated DEGs. The thickness of lines between nodes represents the kappa score of overlapping genes of the linking nodes, calculated by Metascape online tool. $\mathbf{d}$ The top 1 term (ranked by false discovery rate (FDR) q-value) in GSEA enrichment of Control versus PKAi mice, running with the hallmark gene sets of Molecular Signatures Database (MSigDB). The top portion of the plot shows the enrichment scores (ES) for each gene, whereas the bottom portion of the plot shows the value of the ranking metric moving down the list of ranked genes. NES: normalized enrichment score; FWER $p$ value: family wise-error rate. $(N=3)$

The top 10 GO terms, ranked by q-value, were shown in Fig. 2b. Notably, lots of metabolic-associated GO terms (such as "lipid metabolic process", "fatty acid metabolic process" and "cellular metabolic process") were in these DEGs. In PKAi liver, there was increased expression of genes involved in protein stability. Furthermore, the pathway enrichment of the DEGs based on KEGG and Reactome database showed that the "PPAR signaling pathway", was the most significantly enriched pathway in PKAi mice (with both the highest rich factor of 0.188 and the lowest q-value of $6.3 \times 10^{-6}$ ) (Fig. 2c). PPAR $\alpha$, an isoform of PPARs family predominantly expressed in the liver, is a key transcriptional factor in promoting lipid metabolism [39]. Its significant enrichment in the down-regulated DEGs suggested that PKAi mice might had the tendency of less fatty acid degradation in the liver. Finally, we performed GSEA with all the genes detected in the RNA-seq to sensitively predict the most significant marker of the PKAi mice. As a result of the GSEA, the top 1 hallmark, ranked by FDR, is the "FATTY ACID METABOLISM" (Fig. 2d).

Together, we compared the transcriptomes of the liver in PKAi and control mice, indicating that PKAi mainly regulates the expression of genes involved in lipid metabolism.

\section{Hepatic-PKA inhibition had no effect on liver lipid accumulation under chow diet}

The transcriptome analysis suggested a tendency of lipid accumulation in PKAi liver. Thus, we measured their body weight, liver index (ratio of liver weight to body weight) and the triglyceride content in liver tissue. However, 16-week old PKAi mice did not show any significant difference in food consumption (Additional file 6), body weight (Fig. 3a), liver index (Fig. 3b), nor triglyceride content of the liver (Fig. 3c) compared to the control
A

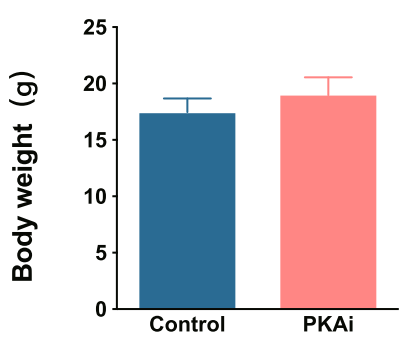

C

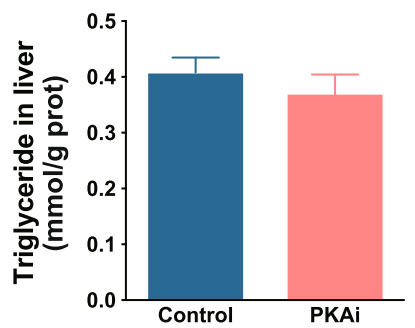

B

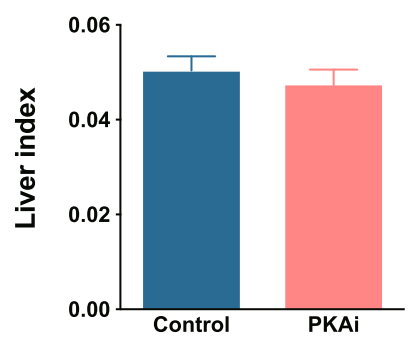

D

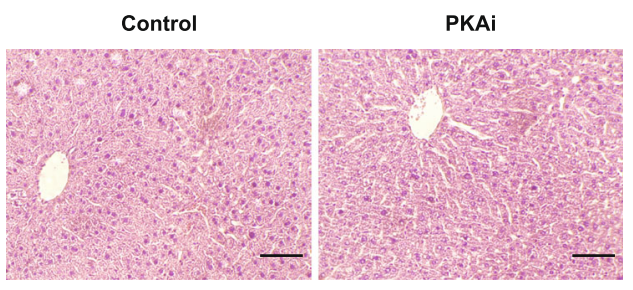

Fig. 3 Hepatic-PKA inhibition had no significant effect on liver lipid accumulation. Mice of 16-week old fed with chow diet (CD) were used. a The body weight of PKAi and control mice. $\mathbf{b}$ The ratio of liver weight to body weight of PKAi and control mice. $\mathbf{c}$ The concentration of triglyceride of the livers. $\mathbf{d}$. The representative images of H\&E staining of liver tissue. Scale bar: $50 \mu \mathrm{m}$. $(N=6)$ 
mice. Further H\&E staining of liver either did not show any significant pathological difference between the two groups (Fig. 3d).

\section{Hepatic-PKA inhibition exacerbated HFD induced lipid accumulation in liver}

As PKAi changes the gene expression involved in lipid metabolism, we further tested lipid metabolism in the liver under metabolic stress in PKAi mice. We fed the PKAi and control mice with HFD for 2 months. Although there was no difference in the food consumption (Additional file 6) and the body weight of PKAi mice compared to control mice (Fig. 4a), the liver weight and the liver index was increased more significantly in PKAi mice (Fig. 4b), which might be attributed to more triglyceride accumulation and hepatocyte enlargement in the liver of PKAi mice (Fig. 4c-e). The accumulation of triglyceride was confirmed by severe hepatocyte vacuolation (Fig. 4d) and increased oil red $\mathrm{O}$ staining (Fig. 4e) in the liver of PKAi mice. Together, the inhibition of PKA activity in vivo aggravated the lipid accumulation in the liver upon HFD stimulation.

\section{HFD feeding differentially altered transcriptomes in control and hepatic-PKAi mice}

Given PKAi mice showed greater lipid accumulation in the liver than the control mice under HFD treatment, next we sought to have a deeper insight of the mechanism and consequences behind this phenomenon. We compared the transcriptome alterations in the liver of control mice or PKAi mice with HFD versus with CD respectively, which enables us to illustrate the different effects of HFD on control mice and PKAi mice.

First, we explored the effects of HFD on the transcriptomes of the liver tissues of control and PKAi mice respectively. In the control mice, 397 genes were upregulated by HFD and 321 genes were down-regulated by HFD (Fig. 5a). GO enrichment analysis (Fig. 5b) showed that inf control mice HFD feeding increased the expression of genes enriched in high-density lipoprotein particle, protein folding, flavonoid metabolism, as well as glucose metabolism (uronic acid, glucuronate metabolism), while the down-regulated genes were enriched in the inflammation/immunity homeostasis. On the other hand, in PKAi mice, 332 genes were up-regulated while 137 genes were down-regulated by HFD (Fig. 5c). Interestingly, the up-regulated genes were enriched in protein folding GO terms, such as "protein folding", "unfolded protein binding", suggesting that severe endoplasmic reticulum (ER) stress was induced by HFD in PKAi mice. However, the down-regulated genes were mainly enriched in cell proliferation and some metabolic processes (terpenoid, diterpenoid, retinoic acid and retinoid). (Fig. 5d).

Then, we compared the transcriptome of liver tissue of control mice and PKAi mice both with HFD

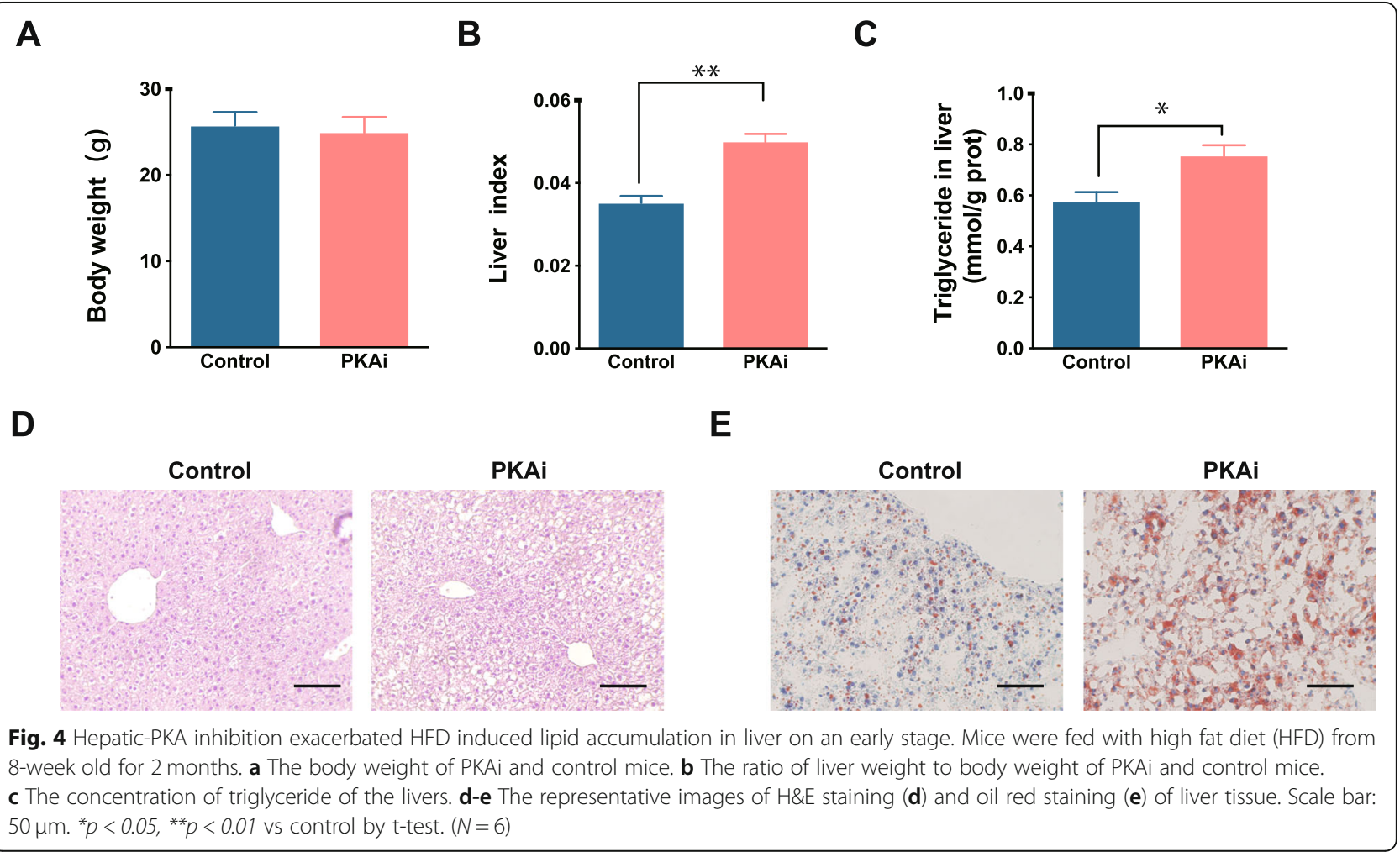




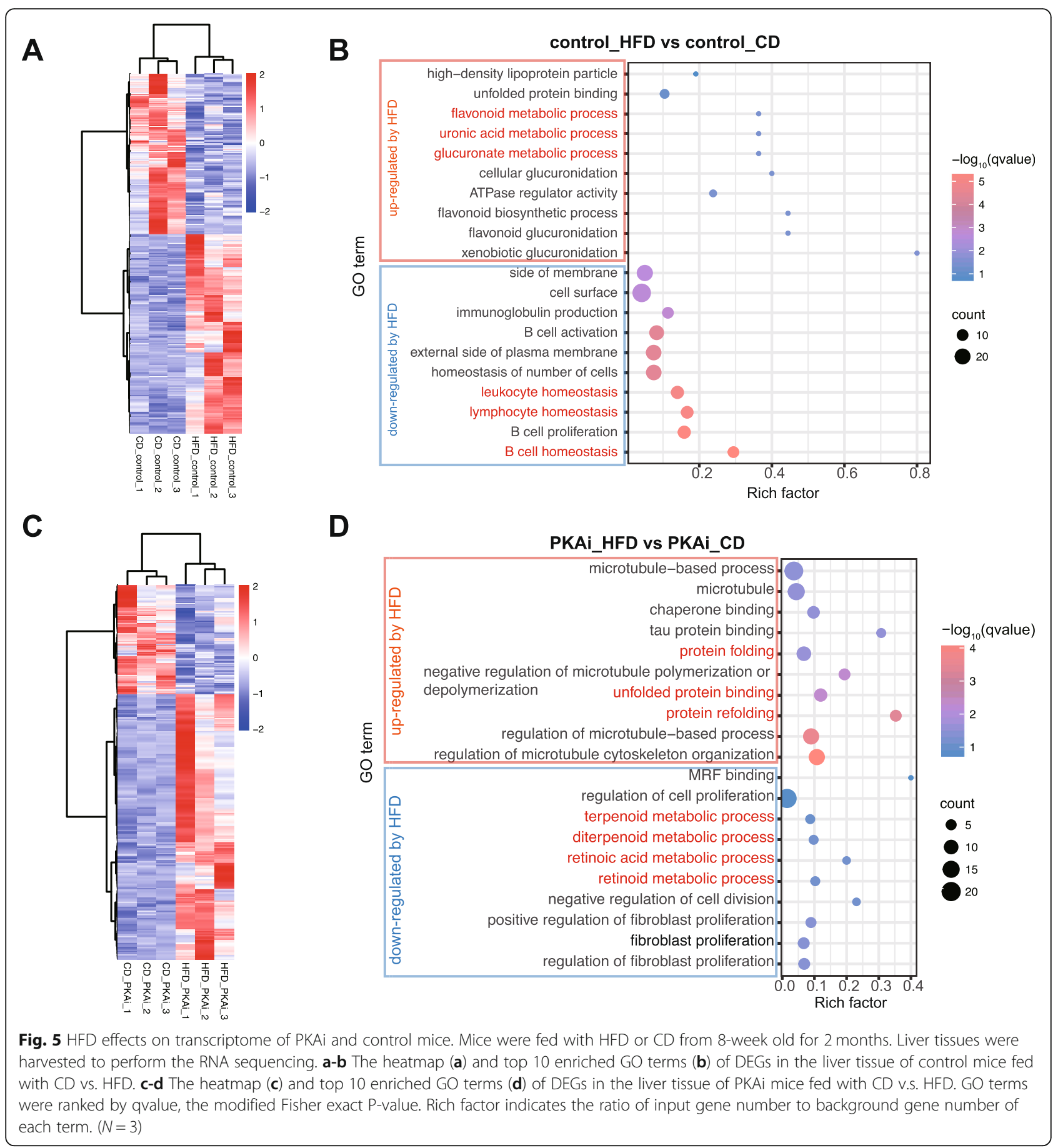

treatment. We found 34 genes were expressed less and 31 gene were expressed more in HFD-induced PKAi mice compared to HFD-induced control mice (Fig. 6a). Further GO analysis of these DEGs (Fig. 6b) showed that genes with lower expression in PKAi_HFD mice (i.e. higher in control mice) were mainly enriched in GO terms with "lipoproteins", which are critical to transport fatty acids, TG and cholesterol out of hepatocytes. This may explain why there was more TG accumulation in
PKAi_HFD mice liver. On the other hand, DEGs with higher expression level in PKAi_HFD mice seemed to be mostly enriched in cell differentiation and immune response.

Finally, we compared the changing directions of DEGs induced by HFD in control and in PKAi mice, which might provide more specific clues to illustrate the different responses to HFD between the two types of mice. By merging the 2 sets of DEGs of the control and PKAi 


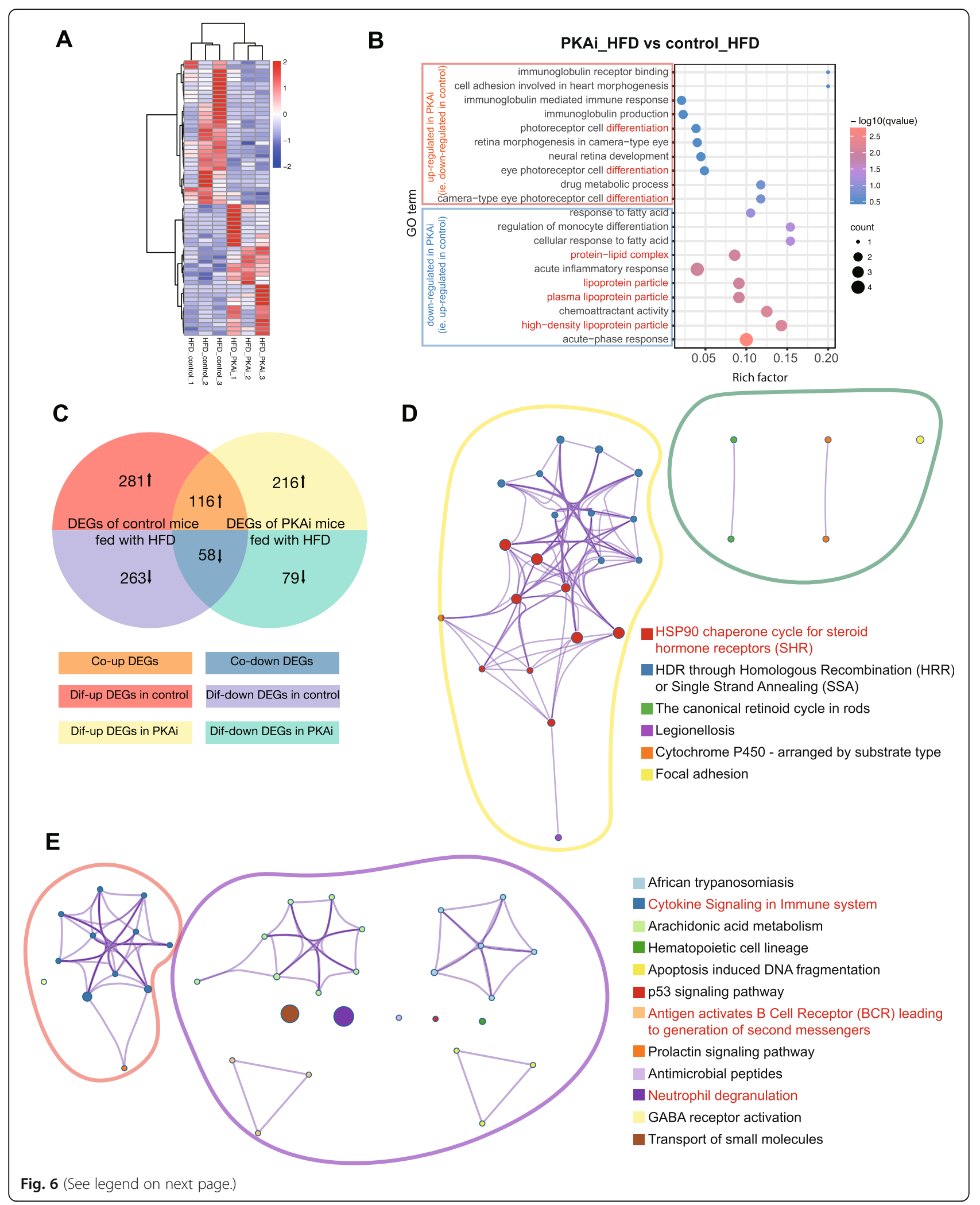


(See figure on previous page.)

Fig. 6 The unique transcriptome alteration of PKAi and control mice induced by HFD. Mice were fed with HFD or CD from 8-week old for 2 months. Liver tissues were harvested to perform the RNA sequencing. $\mathbf{a}-\mathbf{b}$ The heatmap (a) and top 10 enriched GO terms (b) of DEGs in the liver tissue of control mice vs. PKAi mice with HFD feeding. c Venn diagram showing the co-altered or differentially-altered DEGs in PKAi and control mice: orange and blue areas represent co-altered genes, either up- (orange) or down-regulated (blue) on the stimulation of HFD; red and purple areas represent differentially-altered genes in control mice, either up- (red) or down-regulated (purple) on the stimulation of HFD; yellow and green areas represent differentially-altered genes in PKAi mice, either up- (yellow) or down-regulated (green) after the stimulation of HFD. $\mathbf{d}$ e Enriched pathways of differentially-altered genes in PKAi mice (d) and control mice (e). Each node represents a pathway, and the nodes in one color represent a cluster of pathways. The nodes in different-colored circles represent the enriched pathways of DEGs of the same color displayed in $\mathbf{c}$. The thickness of lines between nodes represents the kappa score of overlapping genes of the linking nodes, calculated by Metascape online tool. $(N=3)$

mice effected by HFD (Fig. 6c), we found that 174 genes (termed "co-up/down DEGs" as shown in Fig. 6c) had the same changing direction in both groups (116 upregulated by HFD, 58 down-regulated by HFD). However, 544 and 295 DEGs (termed "dif-up/down DEGs" as shown in Fig. 6c) changed in different directions in control mice (281 up-regulated by HFD, 263 downregulated by HFD) and PKAi mice (216 up-regulated, 79 down-regulated), respectively, which could reflect the different responses to HFD feeding between the two types of mice at the mRNA level. Based on the Reactome and KEGG database, we did pathway enrichment, finding that the unique DEGs in PKAi mice were mostly enriched in "HSP90 chaperone cycle for steroid hormone receptors (SHR)" and "HDR through Homologous Recombination (HRR) or Single Strand Annealing (SSA)", which were closely related to cellular response to stress and DNA repair responding to ER stress (Fig. 6d). On the other hand, the unique DEGs in control mice were mostly enriched in pathways related to immune system, such as "Cytokine Signaling in Immune system", "Antigen activates B Cell Receptor (BCR) leading to generation of second messengers" and "Neutrophil degranulation" (Fig. 6e). These results suggested that HFD caused immune homeostasis alterations in the liver of control mice while activated direct response to stress and DNA repair mechanism in PKAi mice.

\section{Discussion}

In the present study, for the first time we demonstrated that hepatic-PKA inhibition could result in lipid accumulation in the liver, a hallmark of NAFLD [4]. PKAi in hepatocytes not only altered the transcription of genes involved in metabolism of fatty acids and lipids, but also altered the transcriptome response to HFD in the liver. This report provides the first direct evidence of a potential role of PKA in NAFLD development and shed lights on the mechanisms of PKA regulated lipid metabolism in liver physiology and pathology.

As there is no specific PKA inhibitor that can achieve liver-specific PKA inhibition and it is very challenging to make genetic loss-of-function mouse by knocking out all PKA subunits, it has been long sought to determine the role of PKA in liver function in vivo. In this the research area, G. Stan McKnight has constructed many mouse models with single PKA subunit knockout. Zheng et al. reported that disruption of PKA RII $\beta$ subunit in mice adipose tissue leads to a lean phenotype, increased nocturnal locomotor activity, and activation of brown adipose tissue [40]. However, the activity of PKA has not been determined in this study, which might be compensated by RI $\alpha$ at the molecular level. London et al. constructed a ubiquitously ablated PKA RII $\alpha$ mouse model with a phenotype of resistance to diet-induced obesity, glucose intolerance, and hepatic steatosis [41]. But the global deficiency of RII $\alpha$ caused different activities of PKA in some key metabolic organs: cAMP-stimulated PKA activity was decreased in liver but increased in gonadal adipose tissue as reported, which makes it hard to draw the conclusion that the in vivo phenotype is due to the activation or the inhibition of PKA. On the other hand, studies on catalytic subunits also remain ambiguous. Linda et al. constructed a catalytic $C \beta$ global knockout mice, which also protected mice from diet-induced obesity, steatosis, dyslipoproteinemia, and insulin resistance, without any differences in caloric intake or locomotor activity [42]. Theoretically $C \beta$ knockout will lead to a low activity of PKA, which might lead to a decrease of lipolysis as suggested in vitro studies and our results, but a lean phenotype was observed in vivo. This could be due to the compensatory changes of the expression/ activity of other PKA catalytic subunits. Therefore, it is difficult to elucidate the role of PKA in liver physiology and pathology. Here we inhibited PKA activity in a hepatic-specific, controlled by Alb promoter with a double transgenic mouse system to overexpress a PKA inhibiting peptide (PKAi-GFP). In our mouse model, the overexpression of PKAi-GFP successfully inhibited PKA activity in the liver, thus allowing us for the precise and comprehensive study of the roles of PKA in liver tissue. In future studies, it is possible to study the effects of different degrees of PKA inhibitions on the development of NAFLD by screening out transgenic mouse strains with different abundance of PKAi expression, and even achieve the functional PKA ablation model through extremely high expression as reported recently [43]. 
In the present study, the inhibition of PKA activity in the liver showed a normal liver morphology when fed with chow diet and a rapid lipid accumulation in the liver after HFD induction. Our transcriptome study showed considerable differences in the transcriptome of PKAi mice with normal chow feeding, and these differences are largely enriched in lipid metabolism. However, changes in the RNA level appear to be insufficient to cause overt disease phenotype when fed with a control diet. The phenotype could be too subtle or needs more time to develop, such as in ageing mice, which warrants further study. Using HFD as an external stimulus, PKAi mice rapidly developed lipid accumulation in the liver. The development of TG accumulation could be due to multiple reasons: first, our transcriptome analysis suggested that reduced lipid metabolism in hepatocytes of PKAi liver; when fed with HFD chow, PKAi mice had less lipoprotein expression. Lipoproteins play a key role in the transportation of lipids from the liver to peripheral tissues. In this study, some genes related to lipoproteins were up-regulated in control mice when fed with HFD, but were not induced by HFD in PKAi mice, which might account for the less TG accumulation in the liver of control mice. In addition, PKA directly regulates lipase activity in the liver and other metabolic tissues like adipose tissue via phosphorylating these enzymes as reported in the previous in vitro studies [44, 45]. These aspects were not explored in this study to make the study more focused though they need to be studied in the future.

Excessive import or diminished export or oxidation of free fatty acids is the initial step of NAFLD, which activates the immune system to protect the hepatic cells from damage [3]. Once hepatic immune cell is overactivated, inflammation will take place and lead to the NASH. This explains why the immune response genes showed a significant enrichment in control mice after HFD treatment for 8 weeks. In contrast, DEGs in PKAi mice mainly enriched in protective cellular responses to stimuli, including "HSP90 chaperone cycle for steroid hormone receptors (SHR)" and "HDR through Homologous Recombination (HRR) or Single Strand Annealing (SSA)". PKA can be activated by various of hormones, such as catecholamines [46], parathyroid hormone [47], and glucagon [48], and then drives lipolysis. Therefore, inhibition of PKA in the liver may alter the reactivity of the hormones induced response to stimuli. However, one limitation of our study is that we only explored a broad transcriptional alteration by the inhibition of PKA in the liver and provided predictive possible mechanisms, which invokes more experiment-based studies in the future.

In our study, 8 weeks of HFD was sufficient to induce hepatic steatosis in PKAi mice, which is $4-8$ weeks shorter than the time needed for HFD to induce NAFLD in control mice in previous reports [49]. This finding suggests that inhibition of PKA promotes lipid accumulation in the liver, and our PKAi mice fed with HFD can be potentially used as an early stage NAFLD mouse model. It further suggests that reduced PKA activity could be a contributing factor for the development of NAFLD and activating PKA might be a novel strategy to combat the development of NAFLD.

\section{Conclusions}

Together, our data demonstrated that hepatic PKA inhibition alters the lipid metabolism pathways and is sufficient to induce rapid lipid accumulation under HFD treatment.

\section{Supplementary information}

Supplementary information accompanies this paper at https://doi.org/10. 1186/s12986-019-0400-5.

Additional file 1. The formulation of CD and HFD used in the study was demonstrated in the table

Additional file 2. The detail information of the antibodies used in Western blot experiments are listed in the table

Additional file 3. The sequence information of the primers used in qRTPCR are listed in the table

Additional file 4 Total RNA was extracted from different tissues of PKAiGFP mice and GRT-PCR was used to test the PKAi-GFP mRNA expression. ${ }^{* *} p<0.01$ vs liver tissue by one-way ANOVA with with the Tukey-Kramer post hoc test. $(N=3)$

Additional file 5. The gene symbols, fold change and adjusted- $p$ values of the DEGs of PKAi and control mice are listed in the table

Additional file 6. Mice were fed with CD or HFD from 8-week old for 2 months. Twenty-four-hour of food consumption of each group was measured weekly. $(N=6)$

\section{Abbreviations \\ CAMP: cyclic adenosine monophosphate; CD: chow diet; CREB: CAMP response element binding protein; DEG: differentially expressed gene; FDR: false discovery rate; GO: Gene Ontology; GSEA: gene set enrichment analysis; HFD: high-fat diet; HSL: hormone sensitive lipase; KEGG: Kyoto encyclopedia of genes and genomes; MSigDB: Molecular Signatures Database; NAFLD: nonalcoholic fatty liver disease; NASH: nonalcoholic steatohepatitis; PKA: protein kinase A; PKB: protein kinase B; PPARa: peroxisome proliferators-activated receptors alpha; RNF-20: ring finger protein-20; RPKM: reads per kilobase per million mapped reads; SRA: Sequence Read Archive; SREBP-1c: sterol regulatory element binding protein-1c}

\section{Acknowledgements}

The CAG-LoxP-CAT-LoxP-X vector plasmid was povided by Dr. Qinglin Yang at LSU Health New Orleans School of Medicine. L.Y. was supported by the National Heart, Lung, and Blood Institute of the National Institutes of Health under Award Number K22HL139921.

\section{Authors' contributions}

$X C$ designed the study; XYZ cloned the plasmid for making transgenic mice: JNY conducted the experiments; JNY did sample analysis and data analysis, JNY wrote the manuscript; WEW and ZC supported the study; XC, MTM and $L Y$ revised the paper. All authors read and approved the final manuscript. 


\section{Funding}

This study was supported by National Natural Science Foundation of China (grant number: 81673155).

\section{Availability of data and materials}

All data generated or analyzed during this study are included in this published article or are available from the corresponding author on reasonable request.

\section{Ethics approval and consent to participate}

This study was approved by the ethics committee of the Third Military Medical University.

\section{Consent for publication}

The authors consent to the publication of the data.

\section{Competing interests}

The authors declare they have no competing interests.

\section{Author details}

${ }^{1}$ Research Center for Nutrition and Food Safety, Chongqing Key Laboratory of Nutrition and Food Safety, Institute of Military Preventive Medicine, Third Military Medical University, Chongqing, People's Republic of China. ${ }^{2}$ Department of Physiology \& Cardiovascular Research Center, Temple University Lewis Katz School of Medicine, Philadelphia, PA, USA. ${ }^{3}$ Department of Medical Genetics \& Molecular Biochemistry, Temple University Lewis Katz School of Medicine, Room 624 Kresge Hall, Philadelphia, PA, USA. ${ }^{4}$ Daping Hospital, The Third Military Medical University, Chongqing, People's Republic of China.

Received: 19 July 2019 Accepted: 26 September 2019 Published online: 11 October 2019

\section{References}

1. Younossi ZM, Koenig AB, Abdelatif D, Fazel Y, Henry L, Wymer M. Global epidemiology of nonalcoholic fatty liver disease-Meta-analytic assessment of prevalence, incidence, and outcomes. Hepatology (Baltimore, Md). 2016; 64(1):73-84

2. Younossi Z, Anstee QM, Marietti M, Hardy T, Henry L, Eslam M, George J, Bugianesi E. Global burden of NAFLD and NASH: trends, predictions, risk factors and prevention. Nat Rev Gastroenterol Hepatol. 2018;15(1):11-20.

3. Tilg $\mathrm{H}$, Moschen AR. Evolution of inflammation in nonalcoholic fatty liver disease: the multiple parallel hits hypothesis. Hepatology (Baltimore, Md). 2010;52(5):1836-46.

4. Gluchowski NL, Becuwe M, Walther TC, Farese RV Jr. Lipid droplets and liver disease: from basic biology to clinical implications. Nat Rev Gastroenterol Hepatol. 2017;14(6):343-55.

5. Koo SH. Nonalcoholic fatty liver disease: molecular mechanisms for the hepatic steatosis. Clinical and molecular hepatology. 2013;19(3):210-5.

6. Enns LC, Ladiges WC: Targeting PKA Signaling to Prevent Metabolic Syndrome and Delay Aging. In: Medical Complications of Type 2 Diabetes. IntechOpen; 2011.

7. Lu M, Shyy JY. Sterol regulatory element-binding protein 1 is negatively modulated by PKA phosphorylation. Am J Physiol Cell Physiol. 2006;290(6) C1477-86.

8. Zhang Y, Chen ML, Zhou Y, Yi L, Gao YX, Ran L, Chen SH, Zhang T, Zhou X, Zou D, et al. Resveratrol improves hepatic steatosis by inducing autophagy through the CAMP signaling pathway. Mol Nutr Food Res. 2015:59(8):1443-57.

9. Tunaru S, Kero J, Schaub A, Wufka C, Blaukat A, Pfeffer K, Offermanns S. PUMA-G and HM74 are receptors for nicotinic acid and mediate its antilipolytic effect. Nat Med. 2003;9(3):352-5.

10. Yan S, Zhang Q, Zhong X, Tang J, Wang Y, Yu J, Zhou Y, Zhang J, Guo F, Liu $Y$, et al. I prostanoid receptor-mediated inflammatory pathway promotes hepatic gluconeogenesis through activation of PKA and inhibition of AKT. Diabetes. 2014;63(9):2911-23.

11. Miller RA, Chu Q, Xie J, Foretz M, Viollet B, Birnbaum MJ. Biguanides suppress hepatic glucagon signalling by decreasing production of cyclic AMP. Nature. 2013;494(7436):256-60.

12. van Dam AD, Nahon KJ, Kooijman S, van den Berg SM, Kanhai AA, Kikuchi T, Heemskerk MM, van Harmelen V, Lombes M, van den Hoek
AM, et al. Salsalate activates brown adipose tissue in mice. Diabetes. 2015;64(5):1544-54

13. Lee JH, Lee GY, Jang H, Choe SS, Koo SH, Kim JB. Ring finger protein 20 regulates hepatic lipid metabolism through protein kinase A-dependent sterol regulatory element binding protein1c degradation. Hepatology (Baltimore, Md). 2014;60(3):844-57.

14. Yan F, Wang Q, Lu M, Chen W, Song Y, Jing F, Guan Y, Wang L, Lin Y, Bo T, et al. Thyrotropin increases hepatic triglyceride content through upregulation of SREBP-1c activity. J Hepatol. 2014;61(6):1358-64.

15. Hanks SK, Quinn AM, Hunter T. The protein kinase family: conserved features and deduced phylogeny of the catalytic domains. Science (New York, NY). 1988;241(4861):42-52.

16. Knighton DR, Zheng JH, Ten Eyck LF, Xuong NH, Taylor SS, Sowadski JM. Structure of a peptide inhibitor bound to the catalytic subunit of cyclic adenosine monophosphate-dependent protein kinase. Science (New York NY). 1991;253(5018):414-20.

17. Taylor SS, Ilouz R, Zhang P, Kornev AP. Assembly of allosteric macromolecular switches: lessons from PKA. Nat Rev Mol Cell Biol. 2012 13(10):646-58.

18. Steinberg D, Huttunen JK. The role of cyclic AMP in activation of hormonesensitive lipase of adipose tissue. Advances in cyclic nucleotide research 1972;1:47-62.

19. Murray AJ. Pharmacological PKA inhibition: all may not be what it seems. Sci Signal. 2008;1(22):re4.

20. Kim H, Kim M, Im SK, Fang S. Mouse Cre-LoxP system: general principles to determine tissue-specific roles of target genes. Laboratory animal research. 2018;34(4):147-59.

21. Zhang X, Szeto C, Gao E, Tang M, Jin J, Fu Q, Makarewich C, Ai X, Li Y, Tang A, et al. Cardiotoxic and cardioprotective features of chronic betaadrenergic signaling. Circ Res. 2013;112(3):498-509.

22. Glass DB, Cheng HC, Mende-Mueller L, Reed J, Walsh DA. Primary structural determinants essential for potent inhibition of CAMP-dependent protein kinase by inhibitory peptides corresponding to the active portion of the heat-stable inhibitor protein. J Biol Chem. 1989:264(15):8802-10.

23. Kim T, Zhelyabovska O, Liu J, Yang Q. Generation of an inducible, cardiomyocyte-specific transgenic mouse model with PPAR beta/delta overexpression. Methods in molecular biology (Clifton, NJ). 2013;952:57-65.

24. Livak KJ, Schmittgen TD. Analysis of relative gene expression data using real-time quantitative PCR and the $2(-$ Delta Delta $C(T)$ ) method. Methods. 2001;25(4):402-8.

25. Kim D, Langmead B, Salzberg SL. HISAT: a fast spliced aligner with low memory requirements. Nat Methods. 2015;12(4):357-60.

26. Liao Y, Smyth GK, Shi W. featureCounts: an efficient general purpose program for assigning sequence reads to genomic features. Bioinformatics. 2014;30(7):923-30.

27. Robinson MD, McCarthy DJ, Smyth GK. edgeR: a bioconductor package for differential expression analysis of digital gene expression data. Bioinformatics. 2010;26(1):139-40.

28. M P: heatmaps: Flexible Heatmaps for Functional Genomics and Sequence Features. R package 2019, version 1.8.0.

29. Xie C, Mao X, Huang J, Ding Y, Wu J, Dong S, Kong L, Gao G, Li CY, Wei L: KOBAS 2.0: a web server for annotation and identification of enriched pathways and diseases. Nucleic Acids Res 2011, 39(Web Server issue):W316W322.

30. Ginestet CJJotRSSSA: ggplot2: elegant graphics for data analysis. 2011 , 174(1):245-246.

31. Subramanian A, Tamayo P, Mootha VK, Mukherjee S, Ebert BL, Gillette MA, Paulovich A, Pomeroy SL, Golub TR, Lander ES, et al. Gene set enrichment analysis: a knowledge-based approach for interpreting genome-wide expression profiles. Proc Natl Acad Sci U S A. 2005;102(43):15545-50.

32. Liberzon A, Birger C, Thorvaldsdottir H, Ghandi M, Mesirov JP, Tamayo P. The molecular signatures database (MSigDB) hallmark gene set collection. Cell Syst. 2015;1(6):417-25

33. Zhou Y, Zhou B, Pache L, Chang M, Khodabakhshi AH, Tanaseichuk O, Benner C, Chanda SK. Metascape provides a biologist-oriented resource for the analysis of systems-level datasets. Nat Commun. 2019;10(1):1523.

34. Croft D, O'Kelly G, Wu G, Haw R, Gillespie M, Matthews L, Caudy M, Garapati P, Gopinath G, Jassal B, et al. Reactome: a database of reactions, pathways and biological processes. Nucleic Acids Res. 2011;39(Database issue):D691-7.

35. Kanehisa M, Goto SJ Nar: KEGG: kyoto encyclopedia of genes and genomes. 2000, 28(1):27-30 
36. Zeng X, Yang J, Hu O, Huang J, Ran L, Chen M, Zhang Y, Zhou X, Zhu J, Zhang $Q$, et al. Dihydromyricetin ameliorates nonalcoholic fatty liver disease by improving mitochondrial respiratory capacity and redox homeostasis through modulation of SIRT3 signaling. Antioxid Redox Signal. 2018.

37. Yang H, Yang L. Targeting CAMP/PKA pathway for glycemic control and type 2 diabetes therapy. J Mol Endocrinol. 2016;57(2):R93-r108.

38. Fisher FM, Maratos-Flier E. Understanding the physiology of FGF21. Annu Rev Physiol. 2016;78:223-41.

39. Liss KH, Finck BN. PPARs and nonalcoholic fatty liver disease. Biochimie. 2017;136:65-74.

40. Zheng R, Yang L, Sikorski MA, Enns LC, Czyzyk TA, Ladiges WC, McKnight GS. Deficiency of the Rllbeta subunit of PKA affects locomotor activity and energy homeostasis in distinct neuronal populations. Proc Natl Acad Sci U S A. 2013;110(17):E1631-40.

41. London E, Nesterova M, Sinaii N, Szarek E, Chanturiya T, Mastroyannis SA, Gavrilova O, Stratakis CA. Differentially regulated protein kinase a (PKA) activity in adipose tissue and liver is associated with resistance to dietinduced obesity and glucose intolerance in mice that lack PKA regulatory subunit type llalpha. Endocrinology. 2014;155(9):3397-408.

42. Enns LC, Morton JF, Mangalindan RS, McKnight GS, Schwartz MW, Kaeberlein MR, Kennedy BK, Rabinovitch PS, Ladiges WC. Attenuation of age-related metabolic dysfunction in mice with a targeted disruption of the Cbeta subunit of protein kinase a. J Gerontol A Biol Sci Med Sci. 2009;64(12): 1221-31.

43. Zhang Y, Wang WE, Zhang X, Li Y, Chen B, Liu C, Ai X, Zhang X, Tian Y, Zhang $C$, et al. Cardiomyocyte PKA ablation enhances basal contractility while eliminates cardiac beta-adrenergic response without adverse effects on the heart. Circ Res. 2019;124(12):1760-77.

44. Brasaemle DL, Subramanian V, Garcia A, Marcinkiewicz A, Rothenberg A. Perilipin a and the control of triacylglycerol metabolism. Mol Cell Biochem. 2009;326(1-2):15-21.

45. Anthonsen MW, Ronnstrand L, Wernstedt C, Degerman E, Holm C. Identification of novel phosphorylation sites in hormone-sensitive lipase that are phosphorylated in response to isoproterenol and govern activation properties in vitro. J Biol Chem. 1998:273(1):215-21.

46. Schott MB, Rasineni K, Weller SG, Schulze RJ, Sletten AC, Casey CA, MA MN. beta-adrenergic induction of lipolysis in hepatocytes is inhibited by ethanol exposure. J Biol Chem. 2017;292(28):11815-28.

47. Larsson S, Jones HA, Goransson O, Degerman E, Holm C. Parathyroid hormone induces adipocyte lipolysis via PKA-mediated phosphorylation of hormone-sensitive lipase. Cell Signal. 2016;28(3):204-13.

48. Geisler CE, Renquist BJ. Hepatic lipid accumulation: cause and consequence of dysregulated glucoregulatory hormones. J Endocrinol. 2017;234(1):R1-r21.

49. Stephenson K, Kennedy L, Hargrove L, Demieville J, Thomson J, Alpini G, Francis H. Updates on dietary models of nonalcoholic fatty liver disease: current studies and insights. Gene Expr. 2018;18(1):5-17.

\section{Publisher's Note}

Springer Nature remains neutral with regard to jurisdictional claims in published maps and institutional affiliations.

Ready to submit your research? Choose BMC and benefit from:

- fast, convenient online submission

- thorough peer review by experienced researchers in your field

- rapid publication on acceptance

- support for research data, including large and complex data types

- gold Open Access which fosters wider collaboration and increased citations

- maximum visibility for your research: over $100 \mathrm{M}$ website views per year

At $\mathrm{BMC}$, research is always in progress.

Learn more biomedcentral.com/submissions 